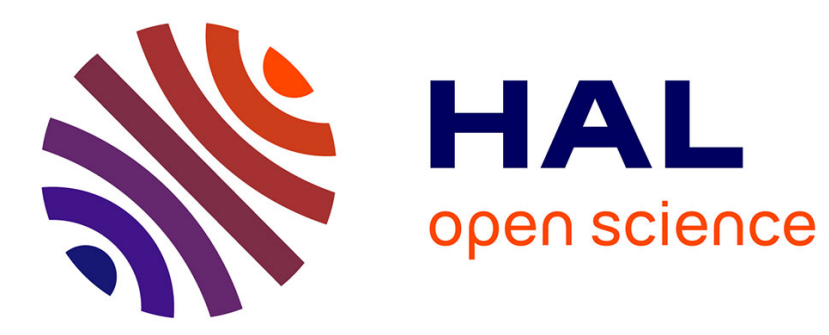

\title{
Robust Detection of Binary CPMs With Unknown Modulation Index
}

Malek Messai, Colavolpe Giulio, Karine Amis Cavalec, Frédéric Guilloud

\section{To cite this version:}

Malek Messai, Colavolpe Giulio, Karine Amis Cavalec, Frédéric Guilloud. Robust Detection of Binary CPMs With Unknown Modulation Index. IEEE Communications Letters, 2015, 19 (3), pp.339 - 342. 10.1109/LCOMM.2015.2390230 . hal-01201662

\section{HAL Id: hal-01201662 \\ https://hal.science/hal-01201662}

Submitted on 17 Sep 2015

HAL is a multi-disciplinary open access archive for the deposit and dissemination of scientific research documents, whether they are published or not. The documents may come from teaching and research institutions in France or abroad, or from public or private research centers.
L'archive ouverte pluridisciplinaire HAL, est destinée au dépôt et à la diffusion de documents scientifiques de niveau recherche, publiés ou non, émanant des établissements d'enseignement et de recherche français ou étrangers, des laboratoires publics ou privés. 


\title{
Robust Detection of Binary CPMs with Unknown Modulation Index
}

\author{
Malek Messai, Member, IEEE, Giulio Colavolpe, Senior Member, IEEE, Karine Amis, Member, IEEE, \\ and Frédéric Guilloud, Member, IEEE,
}

\begin{abstract}
We consider soft-output detection of a binary continuous phase modulation (CPM) generated through a low-cost transmitter, thus characterized by a significant modulation index uncertainty, and sent over a channel affected by phase noise. The proposed detector is designed by adopting a simplified representation of a binary CPM signal with the principal component of its Laurent decomposition and is obtained by using the framework based on factor graphs and the sum-product algorithm. It does not require an explicit estimation of the modulation index nor of the channel phase and is very robust to large uncertainties of the nominal value of the modulation index. Being soft-output in nature, this detector can be employed for iterative detection/decoding of practical coded schemes based on a serial concatenation, possibly through a pseudo-random interleaver, of an outer encoder and a CPM modulation format.
\end{abstract}

Keywords-Continuous phase modulation, modulation index mismatch, phase noise, factor graph, sum product, iterative detection and decoding.

\section{INTRODUCTION}

Continuous-phase modulations (CPMs) [1] are very interesting modulation formats which combine a constant signal envelope and excellent spectral efficiency properties [2]. In particular, the constant envelope makes these modulations insensitive to nonlinear distortions and thus very attractive for an employ in satellite communications and in low-cost and low-power consumption transmitter standards. An analog implementation of the CPM modulator allows to further reduce the transmitter cost, at the expense of possible variations of the CPM waveform parameters around their nominal values. In particular, the modulation index will vary since it depends on the not well calibrated gain of the employed voltage-controlled oscillator (VCO). As an example, in Bluetooth operating in Basic Rate (BR) and Low Energy (LE) modes, the modulation index is specified to be in the intervals $[0.28,0.35]$ and $[0.45,0.55]$, respectively [3]. The interval of the modulation index for the Digital Enhanced Cordless Telecommunication Ultra Low Energy (DECT ULE) is [0.35, 0.7] [4]. In the Automatic Identification System (AIS), the modulation index is nominally equal to 0.5 but due to the imperfections of the AIS equipments, a variation of $\pm 10 \%$ is typically admitted [5].

Manuscript received November 29, 2014; revised December 26, 2014 and accepted January 05,2015 . The associate editor coordinating the review of this letter and approving it for publication was Jinho Choi.

M. Messai, K. Amis and F. Guilloud are with the Signal and Communication Department of Telecom Bretagne-Institut Telecom, CNRS LabSTICC (UNR 6285), Brest 29200, France (e-mail:malek.messai, karine.amis, frederic.guilloud@telecom-bretagne.eu)

G. Colavolpe is with Università di Parma, Dipartimento di Ingegneria dellInformazione, Viale G. P. Usberti, 181A, I-43124 Parma, Italy, e-mail: giulio@unipr.it.
On the other hand, the optimal maximum a-posteriori (MAP) sequence or symbol detectors for CPMs described in the literature require a perfect knowledge of the modulation index at the receiver. In addition, they can be implemented through the Viterbi or the BCJR algorithm, respectively, only when the CPM signal can be described through a finite-state model, and thus when the modulation index is rational [6]. When the modulation index is unknown at the receiver or irrational, the implementation of the optimal detector is still an open problem although many suboptimal detectors have been proposed.

One possible solution can be the adoption of a noncoherent detector (e.g., see [7], [8] and references therein), due to its robustness to the phase uncertainty induced by the imperfect knowledge on the modulation index. As an example, in [9], a noncoherent receiver for the Gaussian frequency shift keying (GFSK) signal adopted in the Bluetooth standard is proposed. This receiver can tolerate only a relatively small modulation index deviation. Another alternative can be represented by the adoption at the receiver of an algorithm for the estimation of the modulation index [10] coupled with the low-complexity algorithms described in [11] or in [12] which is built for a value of the modulation index different from that used at the receiver and properly compensated by using a per-survivor processing.

We here consider the very general problem of soft-input soft-output (SISO) detection of a binary CPM signal with an unknown modulation index transmitted over a channel with phase noise. We adopt a simplified representation of the CPM signal based on the principal component of its Laurent decomposition [13] and describe the joint a-posteriori probability of the transmitted symbols, the channel phase, and the modulation index through a factor graph (FG) [14]. The sum-product algorithm (SPA) is then advocated to compute the a-posteriori probabilities of the transmitted symbols [14]. Due to the recursive nature of the modulator which also makes the CPM well suited for a concatenation with an outer error code, no pilots are required to bootstrap detection. Being soft-output in nature, the proposed algorithm is a good candidate to be employed in iterative detection and decoding schemes.

The letter is organized as follows. The signal model is described in Section II. The proposed algorithm is derived in Section III. Simulations results are discussed in Section IV, whereas conclusions are drawn in Section V.

\section{System Model}

The complex envelope of a binary CPM signal can be expressed as [1]

$$
s(t, \boldsymbol{a}, h)=\sqrt{\frac{2 E_{b}}{T}} \exp \left\{j 2 \pi h \sum_{n} a_{n} q(t-n T)\right\},
$$


where $E_{b}$ is the energy per information bit, $T$ the bit interval, $h$ the modulation index, the function $q(t)$ is the phase-smoothing response, and its derivative is the frequency pulse, assumed of duration $L T$. The information bits $\boldsymbol{a}=\left\{a_{n}\right\}$, assumed independent, belong to the alphabet $\{ \pm 1\}$.

Based on Laurent representation, the complex envelope of the CPM signal may be exactly expressed as [13]

$$
s(t, \boldsymbol{a}, h)=\sum_{k=0}^{K-1} \sum_{n} \alpha_{k, n} p_{k}(t-n T)
$$

where $K=2^{(L-1)}$ and the expressions of pulses $\left\{p_{k}(t)\right\}$ and those of symbols $\left\{\alpha_{k, n}\right\}$ as a function of the information symbol sequence $\left\{a_{n}\right\}$ may be found in [13]. By truncating the summation in (2) considering only the first $K<2^{(L-1)}$ terms, we obtain an approximation of $s(t, \boldsymbol{a}, h)$. Most of the signal power is concentrated in the first component, i.e., that associated with the pulse $\left\{p_{0}(t)\right\}$, which is called principal component [13]. As a consequence, the principal component may be used in (2) to attain a very good trade-off between approximation quality and number of signal components [15], [16]. In this case, it holds

$$
\alpha_{0, n}=\alpha_{0, n-1} e^{j \pi h a_{n}}
$$

When the modulation index is rational, i.e., it can be expressed as $h=r / p$, where $r$ and $p$ are relatively prime integers, symbols $\left\{\alpha_{0, n}\right\}$ take on $p$ values [13].

An important feature of the Laurent decomposition is that the pulse of the principal component weakly depends on the value of the modulation index [10]. This observation will be used for the derivation of the algorithm in Section III. In low cost transmitters, the value of the modulation index is often different from its nominal value which is instead assumed at the receiver. In the following, we will denote by $h_{t x}$ the value of the modulation index at the transmitter and by $h_{r x}$ its nominal value known at the receiver. We also define $h_{e}=h_{t x}-h_{r x}$ as the modulation index mismatch between transmitter and receiver. It is thus $h_{t x}=h_{r x}+h_{e}$ and we will assume that $h_{r x}=r / p$, where $r$ and $p$ are relatively prime integers. On the contrary, $h_{e}$ is assumed unknown and modeled as a random variable with known distribution. We consider transmission over an additive white Gaussian noise (AWGN) channel possibly affected by phase noise. The complex envelope of the received signal thus reads

$$
r(t)=e^{j \theta(t)} s\left(t, \boldsymbol{a}, h_{r x}+h_{e}\right)+w(t)
$$

where $w(t)$ is a complex-valued white Gaussian noise process with independent components, each with two-sided power spectral density $N_{0}$, and $\theta(t)$ is the phase noise introduced by the channel, modeled as a continuous-time Wiener process with incremental variance over a signaling interval equal to $\sigma_{\Delta}^{2}$.

\section{PROPOSED RECEIVER}

Approximating the useful signal through its principal component only and exploiting the feature that the pulse of the principal component weakly depends on the modulation index, we can express the received signal as

$$
r(t) \bumpeq e^{j \theta(t)} \sum_{n} \alpha_{0, n}^{\left(h_{r x}\right)} \alpha_{0, n}^{\left(h_{e}\right)} \hat{p}_{0}(t-n T)+w(t),
$$

where we denoted by $\hat{p}_{0}(t)$ the pulse of the principal component for the nominal value of the modulation index $\left(h_{r x}\right)$ and having exploited the property, easily derived from (3), that symbol $\alpha_{0, n}$ related to the transmitted signal can be expressed as the product of symbol $\alpha_{0, n}^{\left(h_{r x}\right)}$ corresponding to the nominal CPM signal and symbol $\alpha_{0, n}^{\left(h_{e}\right)}$ corresponding to a CPM signal with modulation index $h_{e}$.

Under the assumption that the channel phase $\theta(t)$ is slowly varying such that it can be considered constant over the duration of pulse $\hat{p}_{0}(t)$, an approximate sufficient statistic may be obtained through a filter matched to pulse $\hat{p}_{0}(t)$. We will define

$$
x_{n}=\int_{-\infty}^{+\infty} r(t) \hat{p}_{0}(t-n T) d t
$$

as the output, sampled at time $n T$, of the filter matched to $\hat{p}_{0}(t)$. Defining also $\theta_{n}=\theta(n T), \phi_{n}=\arg \left[\alpha_{0, n}^{\left(h_{r x}\right)}\right]$, $\delta_{n}=\arg \left[\alpha_{0, n}^{\left(h_{e}\right)}\right]$, and $\psi_{n}=\theta_{n}+\phi_{n}+\delta_{n}$, collecting the samples of $\psi_{n}$ into a vector $\boldsymbol{\psi}$, and representing the received signal onto an orthonormal basis and denoting by $\boldsymbol{r}$ its vector representation, we can express, exploiting the constant envelope property of any CPM signal, [16]

$$
p(\boldsymbol{r} \mid \psi) \approx \prod_{n} G_{n}\left(\psi_{n}\right)
$$

with

$$
G_{n}\left(\psi_{n}\right)=\exp \left\{\frac{1}{N_{0}} \operatorname{Re}\left[x_{n} e^{-\jmath \psi_{n}}\right]\right\} .
$$

Samples $\theta_{n}$ satisfy the discrete-time Wiener model

$$
\theta_{n}=\theta_{n-1}+\Delta_{n}
$$

where $\left\{\Delta_{n}\right\}$ are real, independent, and identically distributed Gaussian random variables, with mean zero and variance $\sigma_{\Delta}^{2}$, and $\theta_{0}$ is assumed uniformly distributed in $[0,2 \pi)$. As far as the stochastic model for $h_{e}$, it depends on the considered standard. As an example, in the case of Bluetooth BR [17] $h_{e}$ follows a uniform distribution in the interval $\left[0.28-h_{r x}, 0.35-h_{r x}\right]$, whereas in the case of the AIS standard [5], it follows a Gaussian distribution with mean $0.5-h_{r x}$ and variance $\sigma_{h_{e}}^{2}$.

It is thus

$$
\begin{aligned}
\psi_{n} & =\theta_{n}+\phi_{n}+\delta_{n} \\
& =\theta_{n-1}+\Delta_{n}+\phi_{n-1}+\pi h_{r x} a_{n}+\delta_{n-1}+\pi h_{e} a_{n} \\
& =\psi_{n-1}+\Delta_{n}+\pi h_{r x} a_{n}+\pi h_{e} a_{n} .
\end{aligned}
$$

Assuming that $h_{e}$ has mean zero (this is always possible by properly choosing $h_{r x}$ ) and an even probability density function (pdf), given $a_{n}$ the random variable $\pi h_{e} a_{n}$ is statistically equivalent to $\pi h_{e}$. We will also assume that the random variables $y_{n}=\Delta_{n}+\pi h_{e}$ are independent, ${ }^{1}$ although this is clearly not true. Thus, we may approximate

$$
\begin{aligned}
p(\boldsymbol{\psi} \mid \boldsymbol{a}) & \simeq \prod_{n} p\left(\psi_{n} \mid \psi_{n-1}, a_{n}\right) \\
& =\prod_{n} H_{n}\left(a_{n}, \psi_{n-1}, \psi_{n}\right)
\end{aligned}
$$

\footnotetext{
${ }^{1}$ This assumption corresponds to the case when $h_{e}$ can assume independent values in different symbol intervals.
} 


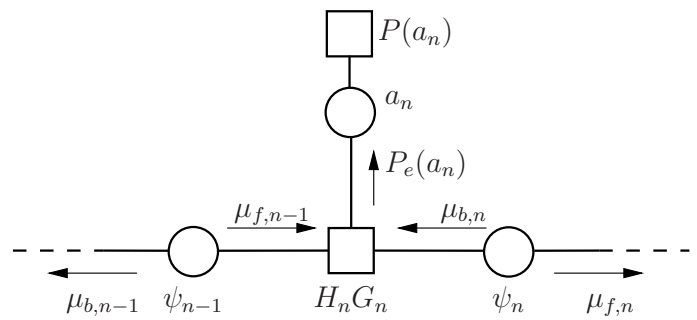

Fig. 1. Portion of the FG corresponding to equation (14).

where

$$
H_{n}\left(a_{n}, \psi_{n-1}, \psi_{n}\right)=f\left(\psi_{n}-\psi_{n-1}-\pi h_{r x} a_{n}\right)
$$

being $f\left(y_{n}\right)$ the pdf of the random variable $y_{n}$ that can be easily computed from the a-priori information available at the receiver (i.e., the distributions of $\Delta_{n}$ and $h_{e}$ and the fact that they are independent). As an example, when $h_{e}$ has uniform distribution in $[-\epsilon, \epsilon]$, we have

$$
f\left(y_{n}\right)=\frac{1}{2 \pi \epsilon}\left[\mathrm{Q}\left(\frac{y_{n}-\pi \epsilon}{\sigma_{\Delta}}\right)-\mathrm{Q}\left(\frac{y_{n}+\pi \epsilon}{\sigma_{\Delta}}\right)\right]
$$

where $\mathrm{Q}(x)=\frac{1}{\sqrt{2 \pi}} \int_{x}^{+\infty} e^{-\frac{u^{2}}{2}} d u$.

We are now ready to derive the optimal MAP symbol detection strategy. The a-posteriori joint distribution of vectors $\boldsymbol{a}$ and $\boldsymbol{\psi}$ can be expressed as

$$
\begin{aligned}
p(\boldsymbol{a}, \boldsymbol{\psi} \mid \boldsymbol{r}) & \propto p(\boldsymbol{r} \mid \boldsymbol{\psi}) p(\boldsymbol{\psi} \mid \boldsymbol{a}) P(\boldsymbol{a}) \\
& \simeq \prod_{n} G_{n}\left(\psi_{n}\right) H_{n}\left(a_{n}, \psi_{n-1}, \psi_{n}\right) P\left(a_{n}\right) .
\end{aligned}
$$

This joint distribution can be represented through a FG. One section of it is shown in Fig. 1.

We can observe that it is cycle-free. Hence, the application to it of the SPA with a non-iterative forward-backward schedule, produces the exact marginal a-posteriori probabilities (APPs) of bits $a_{n}$ (except for the approximation related to the use of the principal components only). With reference to the messages in the figure, by applying the updating rules of the SPA, messages $\mu_{f, n}\left(\psi_{n}\right)$ and $\mu_{b, n}\left(\psi_{n}\right)$ can be recursively computed by means of the following forward and backward recursions:

$$
\begin{gathered}
\mu_{f, n}\left(\psi_{n}\right)=\sum_{a_{n}} P\left(a_{n}\right) \int \mu_{f, n-1}\left(\psi_{n-1}\right) H_{n}\left(a_{n}, \psi_{n-1}, \psi_{n}\right) \\
\cdot G_{n}\left(\psi_{n}\right) d \psi_{n-1} \\
\mu_{b, n-1}\left(\psi_{n-1}\right)=\sum_{a_{n}} P\left(a_{n}\right) \int \mu_{b, n}\left(\psi_{n}\right) H_{n}\left(a_{n}, \psi_{n-1}, \psi_{n}\right) \\
\cdot G_{n}\left(\psi_{n}\right) d \psi_{n}
\end{gathered}
$$

The extrinsic APPs of bits $\left\{a_{n}\right\}$, i.e., $P_{e}\left(a_{n}\right)=$ $P\left(a_{n} \boldsymbol{r}\right) / P\left(a_{n}\right)$ can be finally computed as

$$
\begin{aligned}
P_{e}\left(a_{n}\right) & =\iint \mu_{f, n-1}\left(\psi_{n-1}\right) \mu_{b, n}\left(\psi_{n}\right) \\
& \cdot H_{n}\left(a_{n}, \psi_{n-1}, \psi_{n}\right) G_{n}\left(\psi_{n}\right) d \psi_{n-1} d \psi_{n}
\end{aligned}
$$

This strategy involves integration and computation of continuous pdfs, and it is not suited for direct implementation.
A solution for this problem consists of the use of canonical distributions, i.e., the pdfs $\mu_{f, n}\left(\psi_{n}\right)$ and $\mu_{b, n}\left(\psi_{n}\right)$ computed by the algorithm are constrained to be in a certain "canonical" family, characterized by some parameterization. Hence, the forward and backward recursions reduce to propagating and updating the parameters of the pdf rather than the pdf itself. A very straightforward solution to implement (15)-(17) is obtained by discretizing the channel phase [16]. In this way, the pdfs $\mu_{f, n}\left(\psi_{n}\right)$ and $\mu_{b, n}\left(\psi_{n}\right)$ become probability mass functions (pmfs) and the integrals in (15)-(17) become summations. When the number $D$ of discretization levels is large enough, the resulting algorithm becomes optimal (in the sense that its performance approaches that of the exact algorithm). Other parameterizations are possible to reduce the receiver complexity but they will not be considered here for a lack of space.

\section{Simulation Results}

The performance of the proposed detector is assessed by computer simulations in terms of bit error rate (BER) versus $E_{b} / N_{0}, E_{b}$ being the received signal energy per information bit. In all cases we used a value $D=20$. No performance improvement has been observed with a larger value of $D$.

We first consider an uncoded binary transmission using the GFSK format described in the Bluetooth standard in BR mode. In this case, the modulation index can take its value in the interval $[0.28,0.35]$, randomly, but we will assume that $h_{t x}=0.28$. At the receiver side, for the proposed detector we considered both cases of $h_{r x}=0.3$ and $h_{r x}=1 / 3$. In Fig. 2, we compare the performance of the proposed algorithm with that of the noncoherent detector in [9]. In this latter case, the modulation index is estimated using an estimation period of $N_{e}=50$ symbols and parameters $\alpha$ and $\beta$ described in [9] have been optimized by simulations. The BER performance is compared for different values of phase noise standard deviation $\sigma_{\Delta}$. We can observe that the proposed algorithm performs better than that in [9] and, when the value of $\sigma_{\Delta}$ is low, it performs quite close to the receiver which perfectly knows the modulation index and the channel phase (also shown in the figure).

We now consider the serial concatenation, through a pseudorandom interleaver of length 2048 bits, of a binary convolutional encoder with generators $(7,5)$ (octal notation), and a binary CPFSK modulation with an irrational transmission modulation index of $h_{t x}=\frac{\pi}{5}$. In this case, we compare, for different values of the phase noise standard deviation, the performance of the detection algorithm here proposed when assuming $h_{r x}=5 / 8$, with that of the algorithm [12] which assumes a perfect knowledge of the channel phase and the modulation index. When the modulation index is irrational, a trellis description is not possible and thus we have no performance reference. For this reason, we also considered the performance of the optimal detector when $h_{t x}=h_{r x}=\frac{5}{8}$ (a value very close to the considered irrational modulation index) and the channel phase is perfectly known at the receiver. For all considered receivers, a number of 12 iterations between detector and decoder is allowed. One may observe that the performance of the proposed algorithm is very close to that in [12] although this latter receiver has a perfect knowledge of $h_{t x}$ and the channel phase. The proposed algorithm is thus able to 


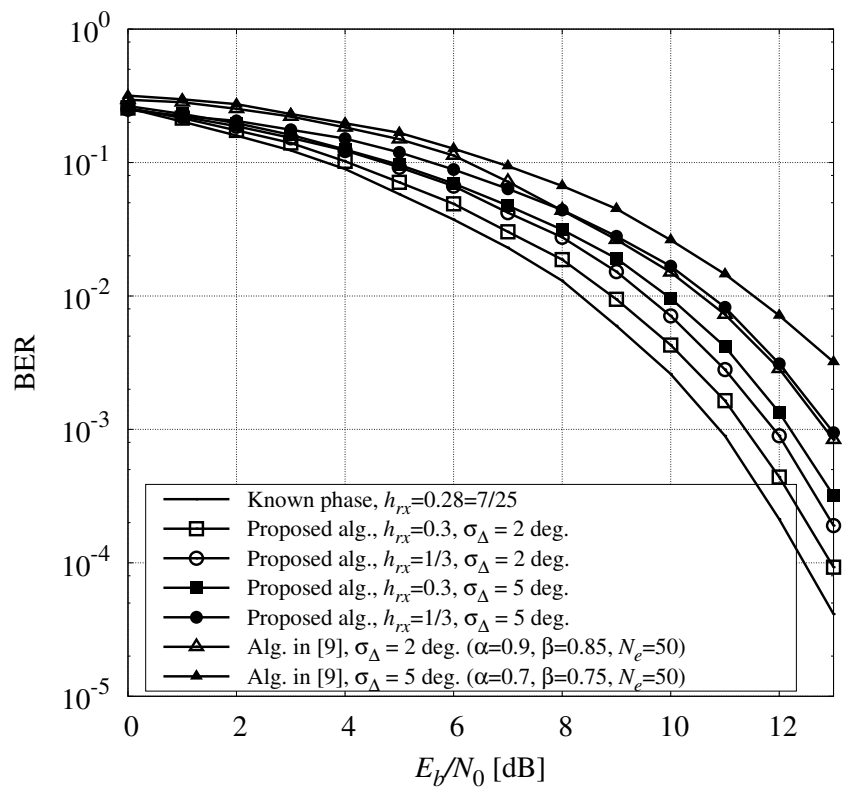

Fig. 2. Bluetooth (BR) detection in the presence of phase noise with standard deviation equal to 2 and 5 degrees.

perform detection even when the modulation index is irrational and not perfectly known and when there is a significant phase noise.

\section{Conclusion}

In this paper, we have derived a robust soft-output detector for binary CPM signals generated through a low-cost transmitter, which leads to a modulation index error, and transmitted over a channel affected by phase noise. The proposed algorithm has been derived based on the Laurent representation of a CPM signal where only the principal component has been considered. Detection in the presence of an unknown modulation index and a time-varying phase noise is performed based on the sum-product algorithm working on a properly defined factor graph. Being soft-output in nature, this detector can be employed for iterative detection/decoding of practical coded schemes based on a serial concatenation, possibly through a pseudo-random interleaver, of an outer encoder and a CPM modulation format.

\section{REFERENCES}

[1] J. B. Anderson, T. Aulin, and C.-E. W. Sundberg, Digital Phase Modulation. New York: Plenum Press, 1986.

[2] A. Barbieri, D. Fertonani, and G. Colavolpe, "Spectrally-efficient continuous phase modulations," IEEE Trans. Wireless Commun., vol. 8, pp. 1564-1572, Mar. 2009.

[3] B. S. I. G. (SIG), "Bluetooth Standard Core Version," 2010.

[4] ESTI, "Digital Enhanced Cordless Telecommunication (DECT); Ultra low Energy (ULE); Machine to Machine Communications; Part 1: Home Automation Network (phase 1)," 2013.

[5] D. Bonacci, J.-P. Millerioux, R. Prevost, J. Lemaitre, M. Coulon, and J.-Y. Tourneret, "Advanced concepts for satellite reception of AIS messages (Toulouse Space Show, Toulouse, 25/06/2012-28/06/2012)," 2012.

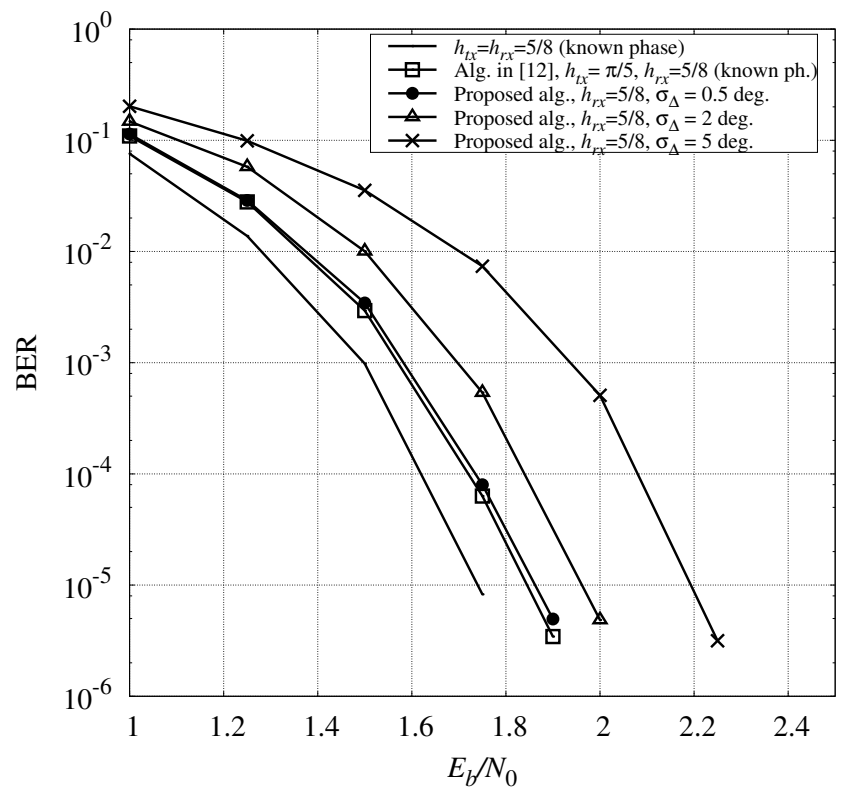

Fig. 3. BER Performance of a binary CPFSK modulation serially concatenated with $\mathrm{CC}(7,5)$ via a pseudo-random interleaver of length 2048 , with irrational modulation index $\frac{\pi}{5}$ in the presence of phase noise with standard deviation $\sigma_{\Delta}$.

[6] B. E. Rimoldi, "A decomposition approach to CPM," IEEE Trans. Inform. Theory, vol. 34, pp. 260-270, Mar. 1988.

[7] G. Colavolpe and R. Raheli, "Noncoherent sequence detection of continuous phase modulations," IEEE Trans. Commun., vol. 47, pp. 1303-1307, Sep. 1999.

[8] L. Lampe, R. Schober, G. Enzner, and J. Huber, "Coded continuous phase modulation with low-complexity noncoherent reception," IEEE Trans. Commun., vol. 50, pp. 517-520, Apr. 2002.

[9] L. Lampe, R. Schober, and M. Jain, "Noncoherent sequence detection receiver for Bluetooth systems," IEEE J. Select. Areas Commun., vol. 23, no. 9, Sep. 2005.

[10] D. Xu and Y. Zhang, "Estimation of the modulation index of CPM signals based on Laurent's decomposition," IEEE Trans. Wireless Commun., vol. 12, no. 12, pp. 6268-6280, Dec. 2013.

[11] M. Messai, F. Guilloud, and K. Amis Cavalec, "A low complexity coherent CPM receiver with modulation index estimation," in Proc. European Signal Processing Conf., 2014.

[12] S. Zarei, W. Gerstacker, G. Kilian, and W. Koch, "An iterative detection algorithm for coded CPFSK signals with irrational modulation index," in Proc. European Signal Processing Conf., Aug. 2012, pp. 2541-2545.

[13] P. A. Laurent, "Exact and approximate construction of digital phase modulations by superposition of amplitude modulated pulses (AMP)," IEEE Trans. Commun., vol. 34, pp. 150-160, Feb. 1986.

[14] F. R. Kschischang, B. J. Frey, and H.-A. Loeliger, "Factor graphs and the sum-product algorithm," IEEE Trans. Inform. Theory, vol. 47, pp. 498-519, Feb. 2001

[15] G. Colavolpe and R. Raheli, "Reduced-complexity detection and phase synchronization of CPM signals," IEEE Trans. Commun., vol. 45, pp. 1070-1079, Sep. 1997.

[16] A. Barbieri and G. Colavolpe, "Simplified soft-output detection of CPM signals over coherent and phase noise channels," IEEE Trans. Wireless Commun., vol. 6, no. 7, pp. 2486-2496, Jul. 2007.

[17] "Specification of the Bluetooth System," http://www.bluetooth.com/, Dec. 2010, Bluetooth Special Interest Group document. 